\title{
AURC Infinity Observed Normalized by
}

\section{Surface Area}

National Cancer Institute

\section{Source}

National Cancer Institute. AURC Infinity Observed Normalized by Surface Area. NCI

Thesaurus. Code C92356.

The area under the urinary excretion rate curve (AURC) extrapolated to infinity, based on the last observed excretion rate, divided by the surface area. 